\title{
Double Percolation Phase Transition in Clustered Complex Networks
}

\author{
Pol Colomer-de-Simón and Marián Boguñá* \\ Departament de Física Fonamental, Universitat de Barcelona, \\ Martí i Franquès 1, 08028 Barcelona, Spain
}

(Received 31 January 2014; revised manuscript received 23 May 2014; published 30 October 2014)

\begin{abstract}
The internal organization of complex networks often has striking consequences on either their response to external perturbations or on their dynamical properties. In addition to small-world and scale-free properties, clustering is the most common topological characteristic observed in many real networked systems. In this paper, we report an extensive numerical study on the effects of clustering on the structural properties of complex networks. Strong clustering in heterogeneous networks induces the emergence of a core-periphery organization that has a critical effect on the percolation properties of the networks. We observe a novel double phase transition with an intermediate phase in which only the core of the network is percolated and a final phase in which the periphery percolates regardless of the core. This result implies breaking of the same symmetry at two different values of the control parameter, in stark contrast to the modern theory of continuous phase transitions. Inspired by this core-periphery organization, we introduce a simple model that allows us to analytically prove that such an anomalous phase transition is, in fact, possible.
\end{abstract}

DOI: 10.1103/PhysRevX.4.041020

\section{INTRODUCTION}

The essence of complex systems lies in the interactions among their constituents. In many cases, these interactions are organized into complex topological architectures that have a determinant role in the behavior and functionality of this class of systems. In regular lattices, dimensionality appears to be one of the most distinctive features; however, randomness and heterogeneity in the interactions of complex networked systems induce phenomena that are very different from, or that are not even observed in, regular lattices. Examples range from the absence of epidemic thresholds that separate healthy and endemic phases [1-6] to the anomalous behavior of Ising-like dynamics [7-10] and percolation properties [11-16].

Percolation theory has played a prominent role in understanding the anomalous behaviors observed in complex networks, and in most cases, it is the common underlying principle behind these behaviors. Interestingly, the interplay between a complex network topology and different percolation mechanisms leads to phenomena that have not previously been observed in statistical physics, including a lack of percolation thresholds in scale-free networks with a degree distribution of the form $P(k) \sim k^{-\gamma}$ for $\gamma<3$ [1-6], anomalous infinite-order percolation transitions in

\footnotetext{
* Corresponding author. marian.boguna@ub.edu

Published by the American Physical Society under the terms of the Creative Commons Attribution 3.0 License. Further distribution of this work must maintain attribution to the author(s) and the published article's title, journal citation, and DOI.
}

Subject Areas: Complex Systems, Statistical Physics

nonequilibrium growing random networks $[17,18]$, or cascading processes in interdependent networks [19-21]. However, these phenomena have already been observed on random graphs with given degree distributions. Random graphs of this type are locally treelike; that is, the number of triangles, and thus the clustering coefficient, can be neglected in the thermodynamic limit. However, the strong presence of triangles is, along with the small-world effect and heterogeneity of the degree distribution, a common and distinctive topological property of many real complex networked systems. While clustering is not a necessary condition for the emergence of any of these phenomena, the effects of clustering on the percolation properties of a network are unknown.

Percolation in clustered networks has been widely studied [22-28]. However, previous reports differ concerning the position of the percolation threshold. Some studies report that clustered networks have a larger percolation threshold than do unclustered networks because of redundant edges in triangles that cannot be used to connect to the giant component (GC) [24-26,28]. Other studies report that strongly clustered networks are more resilient because of the existence of a core that is extremely difficult to break $[22,23,27]$. In fact, as we shall demonstrate, both arguments are correct.

In this paper, we show that strong clustering induces a core-periphery organization in the network [29] that gives rise to a new phenomenon, namely, a "double percolation" transition, in which the core and periphery percolate at different points. This behavior is in stark contrast to the modern theory of continuous phase transitions, which forbids the possibility of breaking the same symmetry at 
(a)

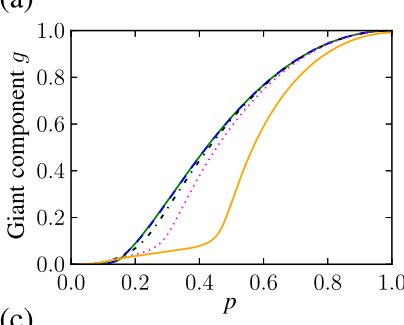

(c)

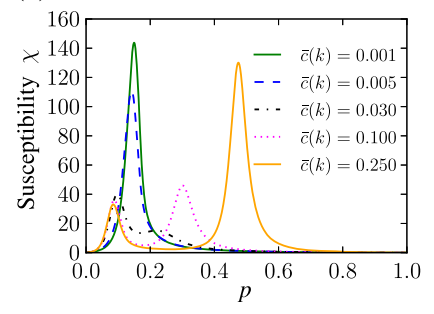

(b)

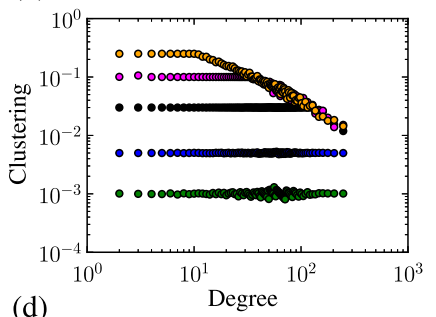

(d)

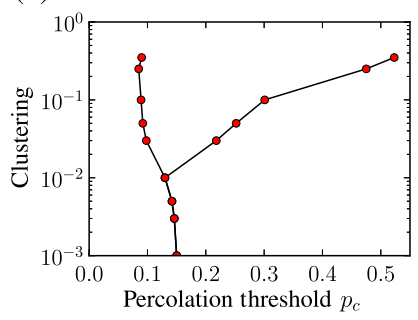

FIG. 1. Bond percolation simulations for networks of $N=$ $5 \times 10^{4}$ nodes with a power-law degree distribution, $\gamma=3.1$, and different levels of clustering. (a) Relative size of the largest connected component $g$ as a function of the bond occupation probability $p$. (b) Degree-dependent clustering coefficient $\bar{c}(k)$. (c) Susceptibility $\chi$ as a function of the bond occupation probability $p$. (d) Percolation threshold $\left(p_{\max }\right)$ as a function of the level of clustering.

two different values of the control parameter. Multiple percolation transitions have recently been reported in Refs. [30-33]. However, in each of these cases, anomalous percolation arises as a consequence of either complex percolation protocols [30-32] or the interdependence between different networks [33], and it is never associated with the same symmetry breaking. Instead, our results are obtained with the simplest percolation mechanism, bond percolation with bond occupation probability $p$, which indicates that this double percolation transition is exclusively induced by a particular organization of the network topology.

\section{RANDOM GRAPHS WITH A GIVEN CLUSTERING SPECTRUM}

We can generate scale-free random graphs with a given clustering spectrum $\bar{c}(k)$ and fixed degree-degree correlations, as shown in Appendix A. A preliminary analysis shows that the percolation properties depend on two network features, the heterogeneity of its degree distribution and the shape of the clustering spectrum $\bar{c}(k)$ [23]. For weakly heterogeneous networks $(\gamma \gg 3)$, we observe that increasing clustering in the network while keeping the degree-degree correlations fixed increases the percolation threshold and decreases the size of the giant component (see Appendix B). However, the most interesting case corresponds to heterogeneous networks, typically with $\gamma<3.5$. In this work, we focus on the case of $\gamma=3.1$ and a constant clustering spectrum [34]. This value of $\gamma$ generates scale-free heterogeneous networks but with a

(a)

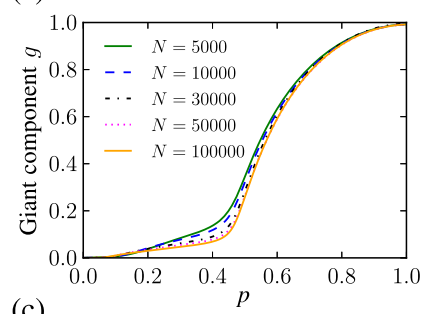

(c)

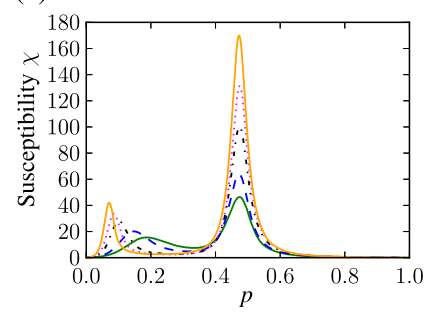

(b)

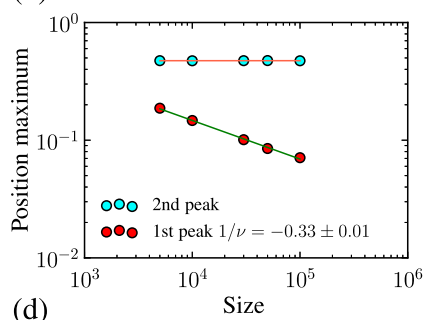

(d)

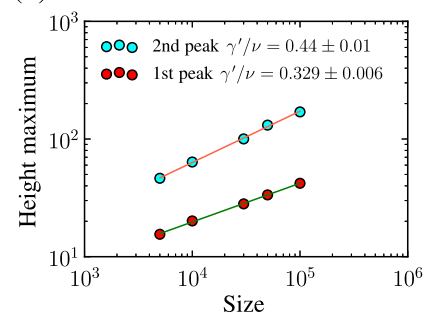

FIG. 2. Bond percolation simulations for networks with a power-law degree distribution with $\gamma=3.1$, target clustering spectrum $\bar{c}(k)=0.25$, and different network sizes. (a) Relative size of the largest connected component as a function of the bond occupation probability $p$. (c) Susceptibility $\chi$ as a function of the bond occupation probability $p$. (b, d) Position $p_{\max }$ and height $\chi_{\max }$ of the two peaks of $\chi$ as functions of the network size $N$. The straight lines are power-law fits, and (b) and (d) show the measured values of the critical exponents.

finite second moment, which allows us to clearly isolate the new phenomenon. The results for $\gamma \leq 3$ are qualitatively similar but more involved and will be presented in a forthcoming publication.

Figure 1 compares the percolation properties of networks with identical degree sequences and degree-degree correlations but with different levels of clustering. For each network, we perform bond percolation $10^{4}$ times using the Newman-Ziff algorithm [36] and measure the average relative size of the largest (giant) connected component, $g \equiv\langle G\rangle / N$, and its fluctuations, i.e., the susceptibility $\chi=\left[\left\langle G^{2}\right\rangle-\langle G\rangle^{2}\right] /\langle G\rangle$. These results are then averaged over 100 network realizations. In finite systems, a peak in the susceptibility $\chi$ indicates the presence of a continuous phase transition, and its position provides an estimate of the percolation threshold (see Appendix E for details). Plots (c) and (d) in Fig. 1 show new and surprising results. For low levels of clustering, there is a unique and well-defined peak in $\chi$, but increasing clustering gives rise to the emergence of a secondary peak at higher values of $p$. This result suggests the presence of a double phase transition, in which two different parts of the network percolate at different times.

To confirm this possibility, we perform finite-size scaling on networks with a target clustering spectrum of $c(k)=$ 0.25 and different system sizes, ranging from $N=5 \times 10^{3}$ to $N=5 \times 10^{5}$. Plot (d) in Fig. 2 shows that the susceptibility exhibits two peaks whose maxima $\chi_{\max }$ diverge as power laws, $\chi_{\max }(N) \sim N^{\gamma^{\prime} / \nu}$ [37]. The position of the first peak also approaches zero as a power law $p_{\max }(N) \sim N^{1 / \nu}$, 
(a)

$\mathrm{O}_{4}$
$\mathrm{O}_{3}$
$\mathrm{O}_{2}$
$\mathrm{O}_{1}$
$\mathrm{O}_{0}$

$\bar{c}(k)=0.001$ (b)

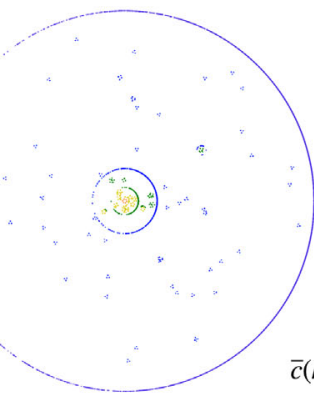

(c)

$\bar{c}(k)=0.25$ (d)

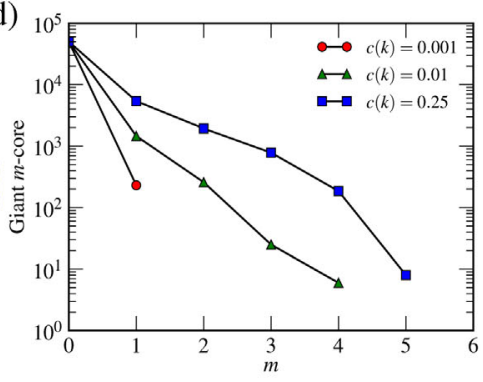

FIG. 3. (a-c) clustering $m$-core decomposition of three different networks with $N=5 \times 10^{4}, \gamma=3.1$, and different levels of clustering, $\bar{c}(k)=0.001,0.1,0.25$. The color code of a node represents its $m$ coreness. For instance, nodes colored violet belong to the $m 0$-core but not to the $m 1$-core and are said to have $m$ coreness of zero. The blue colored nodes belong to the $m 1$-core but not to the $m 2$ core and have $m$ coreness of 1 , etc. The visual representation is as follows. The outermost circle and its contents represent the $m 0$-core and therefore the entire network. If we recursively remove all edges of multiplicity 0 , we obtain the $m 1$-core subgraph, which is contained within the $m 0$-core. Nodes with no remaining connections do not belong to the $m 1$-core, have $m$ coreness of 0 , and are located at the perimeter of the outermost circle. If the $m 1$-core is fragmented into different disconnected components, these components are represented as nonoverlapping circles within the outermost one and with nodes of $m$ coreness of 1 located in their perimeters [see, for instance, panels (b) and (c)]. The same process is repeated for each disconnected $m 1$-core, which will contain a subset of the $m 2$-core, and so on. Links between nodes are not depicted for clarity. (d) The size of the giant $m$-core as a function of $m$ for the networks shown in panels $(\mathrm{a}-\mathrm{c})$.

as shown in Fig. 2(b), which suggests that even if the network has bounded fluctuations, $\left\langle k^{2}\right\rangle<\infty$, it is always percolated in the thermodynamic limit. In contrast, the position of the second peak is nearly constant in the range of sizes we have considered. The divergence of the two peaks in the susceptibility strongly suggests that we are indeed observing two different continuous phase transitions. The first transition is between nonpercolated and percolated phases, and the second transition is between two percolated phases with very different internal organizations.

\section{A. The clustering $\boldsymbol{m}$-core decomposition}

To understand the effect of clustering on the global structure of networks, we use the clustering $m$-core decomposition developed in Ref. [39]. This process is based on the concept of edge multiplicity $m$, which is defined as the number of triangles passing through an edge. We further define the $m$-core as the maximal subgraph whose edges all have at least multiplicity $m$ within it. By increasing $m$ from 0 to $m_{\max }$, we define a set of nested subgraphs that we call the $m$-core decomposition of the network. This decomposition can be represented as a branching process that encodes the fragmentation of $m$-cores into disconnected components as $m$ is increased. The treelike structure of this process provides information regarding the global organization of clustering in networks. To visualize this process, we use the LaNet-vi 3.0 tool developed in Ref. [39] (see the caption of Fig. 3). Figure 3 shows the $m$-core decomposition of three networks with $N=5 \times 10^{4}$ nodes, the same degree sequence (with $\gamma=3.1$ ) and degree-degree correlations, and different levels of clustering. For low levels of clustering, the $m 1$-core is very small, and thus, the $m$-core structure is almost nonexistent. As clustering increases, $m$-cores begin to develop new layers and $m_{\max }$ increases. For instance, for $\bar{c}(k)=0.25$ [Fig. 3(c)], after the recursive removal of all links that do not participate in triangles, we obtain the $m 1$-core that is composed of a large connected cluster with a well-developed internal structure (a core in the center of the figure) and a large number of small disconnected components (a periphery). This result indicates that even if the network is connected, by iteratively removing all edges with multiplicities of zero, we are left with a small but well-connected subgraph, and the reminder of the network is fragmented. Drastic topological transitions induced by clustering have also been reported in the Strauss model and its generalizations [40-42].

The aforementioned result suggests that the two peaks in the susceptibility could be related to this core-periphery organization. Both parts would percolate at different times, first the core and then the periphery, and hence have their own percolation thresholds. To test this hypothesis, we perform bond percolation on the network with a bond occupation probability of $p$ between the two peaks. The giant component at this value of $p$ defines a subgraph that we identify with the core and that roughly corresponds to the core observed in Fig. 3(c) (see Appendix C). We then extract the latter core subgraph from the original network, and the remaining network is thus identified with the periphery. Once the core and periphery are isolated, we perform bond percolation on both components independently and compare the results with the original network. Figure 4 shows that the core percolates precisely at the point where the first peak appears in the original network, whereas the periphery percolates at the second peak. 
(a)

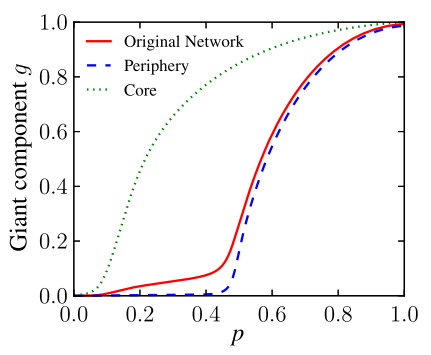

(b)

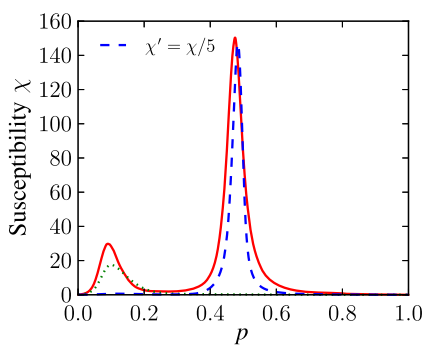

FIG. 4. Bond percolation simulations of the core and periphery of a network with $N=5 \times 10^{4}, \gamma=3.1$, and target clustering spectrum $\bar{c}(k)=0.25$. The bond occupation probability to separate the core is $p=0.2$. The susceptibility curve of the periphery (dashed blue line) has been divided by 5 for ease of comparison.

\section{THE CORE-PERIPHERY RANDOM GRAPH: A SIMPLE MODEL SHOWING A DOUBLE PERCOLATION TRANSITION}

The modern theory of continuous phase transitions states that, in a connected system, it is not possible to break the same symmetry at two different values of the control parameter. In our context, this statement implies that it is not possible to have two genuine percolation transitions at two different values of $p$. It is then unclear whether the second peak observed in our simulations corresponds to a real percolation transition or to a smeared transition, with the percolated core acting as an effective external field that provides connectivity among nodes in the periphery.

Unfortunately, strongly clustered networks cannot be studied analytically. However, we can devise a system with a core-periphery organization similar to that induced by strong clustering. Let us consider two interconnected Erdös-Rényi random graphs, a core and a periphery, of average degrees of $\bar{k}_{c}$ and $\bar{k}_{p}$, respectively. The relative size is $r=N_{c} / N_{p}$, and the average numbers of connections of a node in the core to nodes in the periphery (and vice versa) are $\bar{k}_{c p}$ and $\bar{k}_{p c}=r \bar{k}_{c p}$, respectively. To model a coreperiphery organization, we chose $r<1$ and $\bar{k}_{c}>\bar{k}_{p} \gg \bar{k}_{c p}$. The relative size of the giant component of the combined network is

$$
g(p)=\frac{r}{1+r} g_{c}(p)+\frac{1}{1+r} g_{p}(p),
$$

where $g_{c}(p)$ and $g_{p}(p)$ are the solution of the system of transcendent equations

$$
\left.\begin{array}{r}
g_{c}(p)=1-e^{-p \bar{k}_{c} g_{c}(p)-p \bar{k}_{c p} g_{c p}(p)} \\
g_{c p}(p)=1-e^{-p \bar{k}_{p c} g_{p c}(p)-p \bar{k}_{p} g_{p}(p)} \\
g_{p c}(p)=1-e^{-p \bar{k}_{c p} g_{c p}(p)-p \bar{k}_{c} g_{c}(p)} \\
g_{p}(p)=1-e^{-p \bar{k}_{p} g_{p}(p)-p \bar{k}_{p c} g_{p c}(p)}
\end{array}\right\} .
$$

The derivation of these equations can be found in Appendix D. From here, it readily follows that $g_{c}$ and $g_{p}$ must be either both different from zero or equal to zero, implying that there is generally only one percolation transition, whereas at $p \approx \bar{k}_{p}^{-1}$, there is a crossover effect due to growth of the periphery.

This result is true if the coupling between the core and periphery is macroscopic, that is, if the number of connections between the two structures is proportional to the size of the system such that $\bar{k}_{c p}$ and $\bar{k}_{p c}$ are constants in the thermodynamic limit. Instead, suppose that the number of connections among nodes in the core and periphery scales sublinearly with the system size, i.e., as $N^{\alpha}$ with $0<\alpha<1$. In this case, $\bar{k}_{c p}$ and $\bar{k}_{p c}$ are zero in the thermodynamic limit: Thus, $g_{c}$ and $g_{p}$ become decoupled in Eq. (2) such that $g_{c}$ can be different from zero while $g_{p}=0$. However, when both the core and periphery have a giant connected component as isolated networks, the combined network forms a single connected component because there is an infinite number of connections between each part.

The effect of such structure on bond percolation is as follows. When the bond occupation probability is increased from $p=0$, the first phase transition occurs at $p=\bar{k}_{c}^{-1}$, where the core percolates in a giant component $G_{c} \sim \mathcal{O}(N)$. In the range $\bar{k}_{c}^{-1}<p<\bar{k}_{p}^{-1}$, the periphery is composed of a large number of small disconnected components. The number of such components directly connected to $G_{c}$, and thus the number of nodes in the periphery connected through $G_{c}$, scales as $N^{\alpha}$; therefore, its fraction vanishes in the limit $N \gg 1$, and the relative size of the giant component of the combined system is just $G_{c} / N$. Once we reach $p=\bar{k}_{p}^{-1}$, a percolating cluster is formed in the periphery that becomes macroscopic as we increase $p$ by an infinitesimal amount, i.e., $G_{p} \sim \mathcal{O}(N)$. At this moment, and not before, the number of connections between $G_{c}$ and $G_{p}$ becomes $N^{\alpha-2} G_{c} G_{p} \sim \mathcal{O}\left(N^{\alpha}\right)$ and, consequently, $G_{c}$ and $G_{p}$ are connected with probability 1 . Thus, we have a double percolation transition defined by a regular transition at $p=\bar{k}_{c}^{-1}$ and the sudden emergence at $p=\bar{k}_{p}^{-1}$ of a macroscopic subgraph in the periphery with two types of connectivity; namely, each pair of nodes in this subgraph can be connected not only by a path going through the core but also by a path composed exclusively of nodes outside the core. In turn, this translates into a double discontinuity of the first (or higher) derivative of the order parameter $g$ at $p=\bar{k}_{c}^{-1}$ and $p=\bar{k}_{p}^{-1}$, as clearly seen in Fig. 5(b).

Figures 5(a) and 5(b) present the simulation results of the relative size of the giant component for $\alpha=1$ and $\alpha=0.5$, respectively. In the first case, we observe a crossover effect at approximately $p=\bar{k}_{p}^{-1}$, as also observed in Ref. [43], whereas in the second case, we observe a clear discontinuity in the derivative of $g(p)$ at exactly $p=\bar{k}_{p}^{-1}$, which is consistent with the analytical prediction in Eqs. (1) and (2) for $\bar{k}_{c p}=\bar{k}_{p c}=0$. However, the strongest evidence for the presence of a genuine double phase transition is provided by analysis of the susceptibility. In the case of a crossover effect, fluctuations in the percolated phase behave as 
(a)

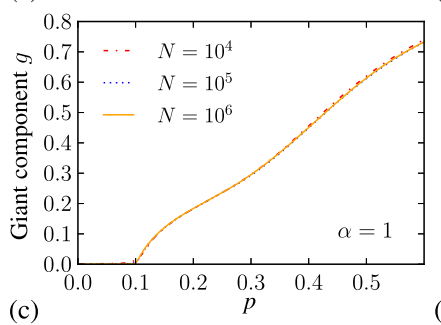

(c)

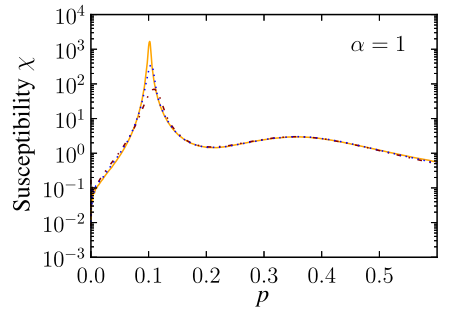

(d)
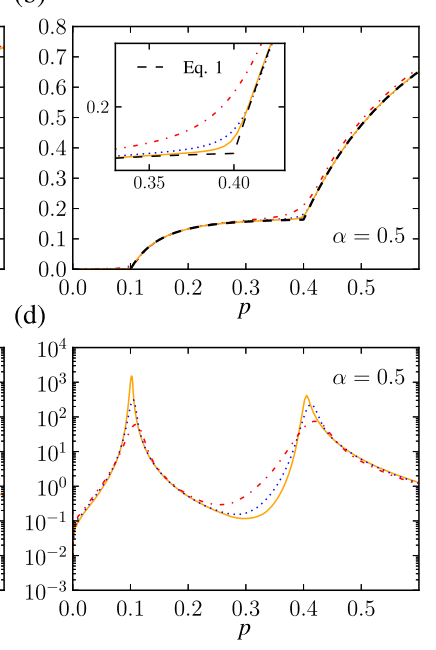

FIG. 5. Bond percolation simulations for the core-periphery random graph model with $\alpha=1$ (left column) and $\alpha=0.5$ (right column). In both cases, the core has an average degree of $\bar{k}_{c}=10$ and the periphery $\bar{k}_{p}=2.5$. The core-periphery ratio is $r=0.2$. The black dashed line in plot (b) is the numerical solution of Eqs. (1) and (2) with $\bar{k}_{c p}=0$. The inset shows the approach to the theoretical prediction at the second transition point as the size of the system is increased.

$\left\langle G^{2}\right\rangle-\langle G\rangle^{2} \sim\langle G\rangle$; consequently, the quantity $\chi$ should diverge at the critical point and become size independent after this point has been surpassed. In contrast, if the second transition in the periphery is a real phase transition, this quantity should diverge at both $p=\bar{k}_{c}^{-1}$ and $p=\bar{k}_{p}^{-1}$. This behavior is clearly observed in Figs. 5(c) and 5(d) (we provide a finite-size analysis of both transitions in Appendix E, Figs. 9 and 10).

In the case of clustered networks, it is difficult to clearly identify the core. Nevertheless, by using the giant $m 1$-core as a rough approximation, we find that, in the case of $\bar{c}(k)=0.25$, the average number of connections between a node not in the giant $m 1$-core and nodes in the giant $m 1$-core is approximately 0.02 , indicating that the core and periphery are, in fact, very weakly coupled. In any case, the double divergence of $\chi$ shown in Fig. 2(c), just as in the core-periphery random graph model with $\alpha<1$, is clear evidence for a genuine double phase transition.

\section{DISCUSSION}

As we have demonstrated, clustering has a nontrivial effect on the properties of complex networks. This effect depends on three main factors: the heterogeneity of the degree distribution, the degree-degree correlations, and the shape of the clustering spectrum $\bar{c}(k)$. If we avoid degreedegree correlations, the combination of strong clustering and heterogeneity induces the emergence of a small but macroscopic core surrounded by a large periphery. This organization redefines the percolation phase space of complex networks by inducing a new percolated phase in which the core of the network is percolated but the periphery is not. In this situation, increasing clustering makes the core larger and more entangled, thereby decreasing the percolation threshold of the first transition, as suggested in Refs. [22,23,27]. However, in the remaining part of the network (the periphery) clustering generates small clique-like structures that are sparsely interconnected [see Fig. 3(c)]. Thus, the periphery becomes more fragile, and the percolation threshold of the second phase transition increases, in agreement with Refs. [24-26,28]. For weakly heterogeneous networks, the size of the core is not macroscopic; thus, clustering only makes these networks more susceptible to the removal of links. This fact reconciles the two dominant interpretations of the effect of clustering on the percolation properties of complex networks. Interestingly, this behavior is also observed in a large sample of real complex networks (see Appendix F), which provides evidence of the generality of this phenomenon.

We have shown that, in contrast to previous theory, it is possible to have two or more consecutive continuous phase transitions associated with the same symmetry breaking. Our work opens new lines of research concerning the effect of this core-periphery architecture on dynamical processes that occur in networks. In the case of epidemic spreading, for instance, the core could act as a reservoir of infectious agents that would be latently active in the core while the remainder of the network is uninfected.

\section{ACKNOWLEDGMENTS}

We thank an anonymous referee for useful comments. This work was supported by the James S. McDonnell Foundation; the ICREA Foundation, funded by the Generalitat de Catalunya; Mineco Projects No. FIS201021781-C02-02 and FIS2013-47282-C2-1-P; Generalitat de Catalunya Grant No. 2014SGR608; and EC FET-Proactive Project MULTIPLEX (Grant No. 317532).

\section{APPENDIX A: MAXIMALLY RANDOM CLUSTERED NETWORKS}

Maximally random clustered networks are generated by means of a biased rewiring procedure similar to the approach used in Refs. [42,44]. One edge is chosen at random that connects nodes $\mathrm{A}$ with $\mathrm{B}$. Then, we choose, at random, a second link attached at least to one node $(\mathrm{C})$ with the same degree of $\mathrm{A}$. This link connects $\mathrm{C}$ with $\mathrm{D}$. Then, the two edges are swapped so that nodes $\mathrm{A}$ and $\mathrm{D}$, on the one hand, and $\mathrm{C}$ and $\mathrm{B}$, on the other, are now connected. We make sure that no self-connections or multiple connection between the same pair of nodes are created in this process. Notice that this procedure preserves both the degree of each node and the actual nodes' degrees at the end of the two original edges. Therefore, the procedure preserves the full degree-degree correlation structure 
encoded in the joint distribution $P\left(k, k^{\prime}\right)$. The procedure is ergodic and satisfies detailed balance.

Regardless of the rewiring scheme used, the process is biased so that generated graphs belong to an exponential ensemble of graphs $\mathcal{G}=\{G\}$, where each graph has a sampling probability $P(G) \propto e^{-\beta H(G)}$, with $\beta$ the inverse of the temperature and $H(G)$ a Hamiltonian that depends on the current network configuration. Here, we consider ensembles where the Hamiltonian depends on the target clustering spectrum $\bar{c}(k)$ as

$$
H=\sum_{k=k_{\min }}^{k_{c}}\left|\bar{c}^{*}(k)-\bar{c}(k)\right|,
$$

where $\bar{c}^{*}(k)$ is the current degree-dependent clustering coefficient. We then use a simulated annealing algorithm based on a standard Metropolis-Hastings procedure. Let $G^{\prime}$ be the new graph obtained after one rewiring event, as defined above. The candidate network $G^{\prime}$ is accepted with probability

$$
p=\min \left(1, e^{\beta\left[H(G)-H\left(G^{\prime}\right)\right]}\right)=\min \left(1, e^{-\beta \Delta H}\right)
$$

(a)

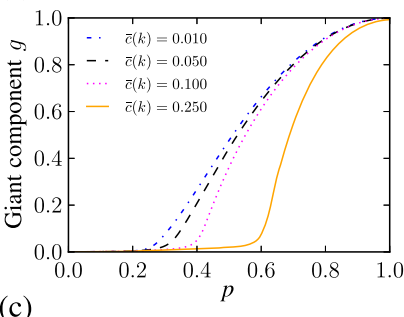

(c)
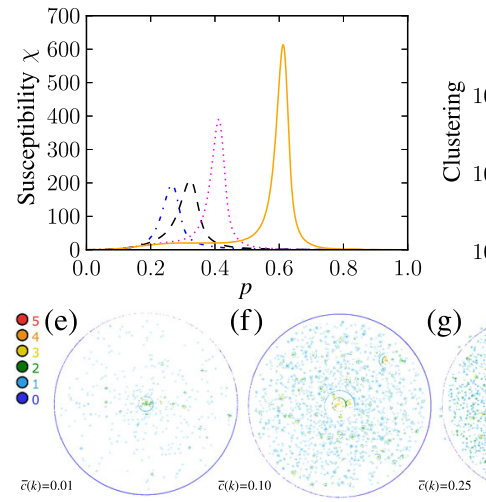

(b)

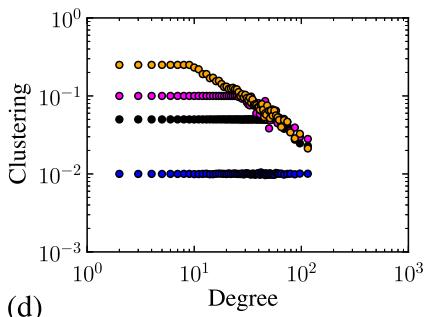

(d)

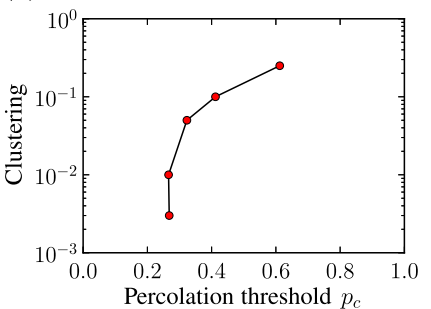

(h)

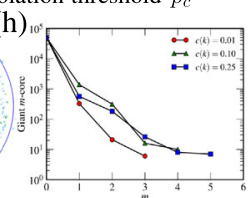

FIG. 6. Top panels: Bond percolation simulations for networks of 10000 nodes with a power-law degree distribution with $\gamma=$ 3.5 and different levels of clustering. (a) Relative size of the largest connected component $g$ as a function of the bond occupation probability $p$. (b) Degree-dependent clustering coefficient $\bar{c}(k)$. (c) Susceptibility $\chi$ as a function of bond occupation probability $p$. (d) Percolation threshold $\left(p_{\max }\right)$ as a function of the level of clustering. Bottom panels: (e-g) $m$-core decomposition of three different networks of 50000 nodes, $\gamma=3.5$, and different levels of clustering, $\bar{c}(k)=0.01,0.10$, 0.25 . (h) Size of the largest connected component of the $m$-core as a function of $m$. otherwise, we keep the graph $G$ unchanged. We first start by rewiring the network $200 E$ times at $\beta=0$, where $E$ is the total number of edges of the network. Then, we start an annealing procedure at $\beta_{0}=50$, increasing the parameter $\beta$ by $10 \%$ after $200 E$ rewiring events have taken place. We keep increasing $\beta$ until the target clustering spectrum is reached within a predefined precision or no further improvement can be achieved.

\section{APPENDIX B: EFFECT OF CLUSTERING ON WEAKLY HETEROGENEOUS NETWORKS}

Figure 6 for $\gamma=3.5$ and Fig. 7 for $\gamma=4$ show the comparison of the percolation properties of networks with exactly the same degree sequence and degree-degree correlations but different levels of clustering. For each network, we perform bond percolation $10^{4}$ times using the Newman-Ziff algorithm [36] and measure the average relative size of the largest (giant) connected component, $g \equiv\langle G\rangle / N$, and its fluctuations, i.e., the susceptibility $\chi=\left[\left\langle G^{2}\right\rangle-\langle G\rangle^{2}\right] /\langle G\rangle$. These results are then averaged over 50 network realizations. In finite systems, a peak in the susceptibility $\chi$ indicates the presence of a continuous (a)

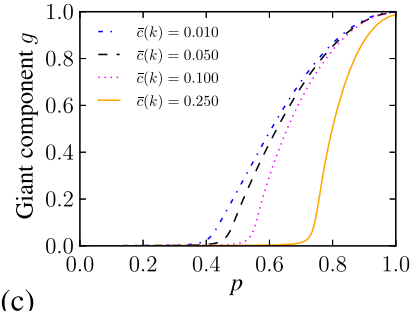

(c)
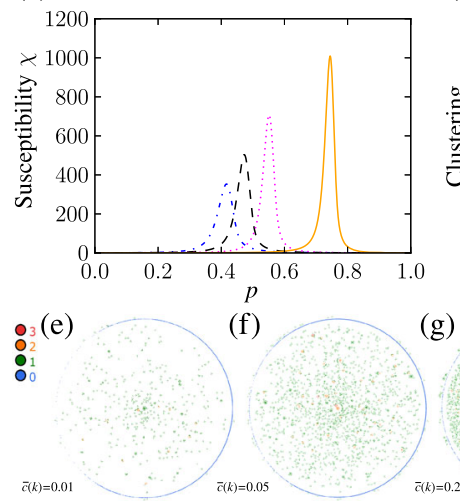

(b)

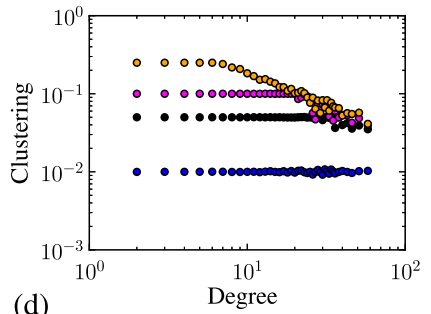

(d)

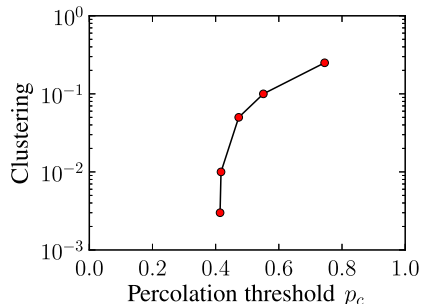

(h)

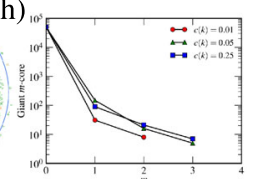

FIG. 7. Top panels: Bond percolation simulations for networks of 10000 with a power-law degree distribution with $\gamma=4$ and different levels of clustering. (a) Relative size of the largest connected component $g$ as a function of the bond occupation probability $p$. (b) Degree-dependent clustering coefficient $\bar{c}(k)$. (c) Susceptibility $\chi$ as a function of bond occupation probability $p$. (d) Percolation threshold $\left(p_{\max }\right)$ as a function of the level of clustering. Bottom panels: (e-g) $m$-core decomposition of three different networks of 50000 nodes, $\gamma=4$, and different levels of clustering, $\bar{c}(k)=0.01,0.05,0.25$. (h) Size of the largest connected component of the $m$-core as a function of $m$. 


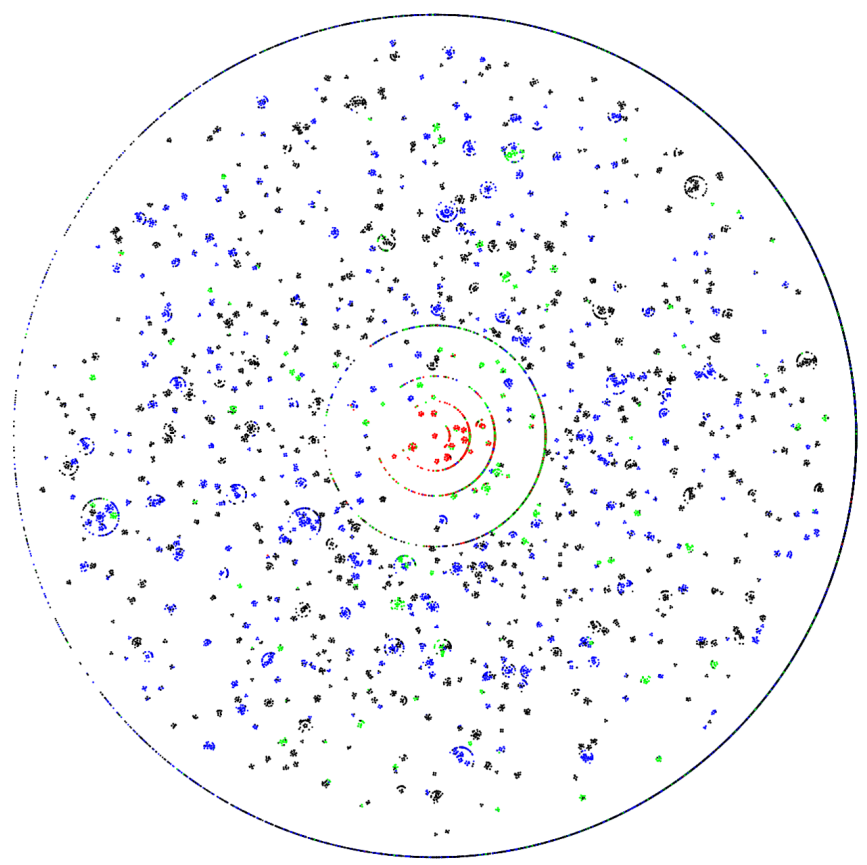

FIG. 8. A network of 50000 nodes, with a power-law degree distribution with $\gamma=3.1$ and a clustering spectrum $\bar{c}(k)=0.25$. The nodes are distributed according to the $m$-core decomposition. Red nodes (1811) are the core because they belong to the giant component after we perform a bond percolation with $p=0.2$ (between the two percolation thresholds). Blue and green nodes are peripheral nodes that belong to the giant component at $p=$ 0.5 (just after the second percolation threshold). Once we subtract the core, blue nodes (10408) still remain in the GC; meanwhile, green nodes (4271) belong to small components. Black nodes (33510) never belong to the GC.

phase transition, and its position gives an estimate of the percolation threshold. All networks have a unique and well-defined peak in $\chi$, and an increase of the clustering moves the peak to higher values of $p$. Hence, clustering decreases the giant component and increases the percolation threshold.

\section{APPENDIX C: IDENTIFICATION OF THE CORE}

In order to identify which nodes belong to the core and which belong to the periphery, we perform a bond percolation simulation on a network of 50000 nodes $\gamma=3.1$ and $c(k)=0.25$. We first delete all edges, and then we add the edges one by one randomly. After we add $20 \%$ of the total number of edges $(p=0.2$ that lays between the two percolation thresholds), the GC defines a subgraph that we identify with the core (red nodes in Fig. 8). If, in the same simulation, we keep adding edges, we will observe another phase transition where the periphery percolates at $p=0.5$. However, the periphery percolates regardless of the core. This can be observed if we subtract the nodes that belong to the core and see that the largest component that remains is still a macroscopic component (blue nodes in Fig. 8), and only a few nodes leave the GC (green nodes in Fig. 8).

\section{APPENDIX D: BOND PERCOLATION ON INTERCONNECTED NETWORKS}

Let us consider two interconnected random graphs $a$ and $b$ with average degrees $\bar{k}_{a a}$ and $\bar{k}_{b b}$, respectively. The relative size is $r=N_{a} / N_{b}$, and the average number of connections of a node in $a$ to nodes in $b$ (and vice versa) are $\bar{k}_{a b}$ and $\bar{k}_{b a}=r \bar{k}_{a b}$. Each node has connections to both networks, and therefore, its degree can be represented as a vector $\vec{k}=\left(k_{a}, k_{b}\right)$. Hence, $P_{a}(\vec{k})$ is the probability of a node of the network $a$ to have degree $\vec{k}$, and $P_{a b}\left(\vec{k}^{\prime} \mid \vec{k}\right)$ is the probability that a node of $a$ with degree $\vec{k}$ is connected to a node of $b$ with degree $\vec{k}^{\prime}$. The relative size of the giant component of the combined network is

$$
g(p)=\frac{r}{1+r} g_{a}(p)+\frac{1}{1+r} g_{b}(p)
$$

where $g_{a}$ is the probability that a node of $a$ belongs to the giant component, or 1 minus the probability that it belongs to a finite cluster, that is, $g_{a}=1-\sum_{s=0}^{\infty} Q_{a}(s)$, where $Q_{a}(s)$ is the probability that a randomly chosen node from network $a$ belongs to a cluster of size $s$.

In heterogeneous networks, the size of the cluster a given node belongs to is correlated with the degree of the node. Thus, $Q_{a}(s)$ must be evaluated as $Q_{a}(s)=$ $\sum_{\vec{k}} P_{a}(\vec{k}) Q_{a}(s \mid \vec{k})$, where $Q_{a}(s \mid \vec{k})$ is the probability that a node from network $a$ of degree $\vec{k}$ belongs to a cluster of size $s$. The latter function satisfies

$$
\begin{aligned}
Q_{a}(s \mid \vec{k})= & \sum_{n_{a}}\left(\begin{array}{c}
k_{a} \\
n
\end{array}\right)_{a} p^{n_{a}}(1-p)^{k_{a}-n_{a}} \sum_{n_{b}}\left(\begin{array}{c}
k_{b} \\
n
\end{array}\right)_{b} p^{n_{b}}(1-p)^{k_{b}-n_{b}} \\
& \sum_{s_{1} \cdots s_{n_{a}}} G_{a a}\left(s_{1} \mid \vec{k}\right) \cdots G_{a a}\left(s_{n_{a}} \mid \vec{k}\right) \sum_{s_{1}^{\prime} \cdots s_{n_{b}}^{\prime}} G_{a b}\left(s_{1}^{\prime} \mid \vec{k}\right) \cdots G_{a b}\left(s_{n_{b}}^{\prime} \mid \vec{k}\right) \\
& \delta_{s, 1+s_{1}+\cdots+s_{n_{a}}+s_{1}^{\prime}+\cdots+s_{n_{b}}^{\prime}},
\end{aligned}
$$

where $G_{a a}(s \mid \vec{k})\left(G_{a b}(s \mid \vec{k})\right)$ is the probability to reach $s$ other nodes by following a neighbor in network $a(b)$. The generating function of $Q_{a}(s \mid \vec{k})$ can be written as 


$$
\hat{Q}_{a}(z \mid \vec{k})=\sum_{s=0}^{\infty} Q_{a}(s \mid \vec{k}) z^{s}=z\left(1-p+p \hat{G}_{a a}(z \mid \vec{k})\right)^{k_{a}}\left(1-p+p \hat{G}_{a b}(z \mid \vec{k})\right)^{k_{b}}
$$

Functions $G_{a a}(s \mid \vec{k}), G_{a b}(s \mid \vec{k}), G_{b a}(s \mid \vec{k})$, and $G_{b b}(s \mid \vec{k})$ follow similar recurrence equations. Thus, their generating functions satisfy

$$
\begin{aligned}
& \hat{G}_{a a}(z \mid \vec{k})=z \sum_{\vec{k}} P_{a a}\left(\vec{k}^{\prime} \mid \vec{k}\right)\left(1-p+p \hat{G}_{a a}(z \mid \vec{k})\right)^{k_{a}^{\prime}-1}\left(1-p+p \hat{G}_{a b}(z \mid \vec{k})\right)^{k_{b}^{\prime}}, \\
& \hat{G}_{a b}(z \mid \vec{k})=z \sum_{\vec{k}} P_{a b}\left(\vec{k}^{\prime} \mid \vec{k}\right)\left(1-p+p \hat{G}_{b a}(z \mid \vec{k})\right)^{k_{a}^{\prime}-1}\left(1-p+p \hat{G}_{b b}(z \mid \vec{k})\right)^{k_{b}^{\prime}}, \\
& \hat{G}_{b a}(z \mid \vec{k})=z \sum_{\vec{k}} P_{b a}\left(\vec{k}^{\prime} \mid \vec{k}\right)\left(1-p+p \hat{G}_{a a}(z \mid \vec{k})\right)^{k_{a}^{\prime}}\left(1-p+p \hat{G}_{a b}(z \mid \vec{k})\right)^{k_{b}^{\prime}-1}, \\
& \hat{G}_{b b}(z \mid \vec{k})=z \sum_{\vec{k}} P_{b b}\left(\vec{k}^{\prime} \mid \vec{k}\right)\left(1-p+p \hat{G}_{b a}(z \mid \vec{k})\right)^{k_{a}^{\prime}}\left(1-p+p \hat{G}_{b b}(z \mid \vec{k})\right)^{k_{b}^{\prime}-1},
\end{aligned}
$$

where $P_{a a}\left(\vec{k}^{\prime} \mid \vec{k}\right)$ is the probability that a randomly chosen neighbor among all the $a$ neighbors of a node that belongs to network $a$ with degree $\vec{k}$ has degree $\vec{k}^{\prime}$, and analogously for the rest of the transition probabilities.

For networks with no degree-degree correlations, these transition probabilities simplify as

$$
\begin{array}{cc}
P_{a a}\left(\vec{k}^{\prime} \mid \vec{k}\right)=\frac{k_{a}^{\prime} P_{a}\left(\vec{k}^{\prime}\right)}{\vec{k}_{a a}} & P_{b b}\left(\vec{k}^{\prime} \mid \vec{k}\right)=\frac{k_{b}^{\prime} P_{b}\left(\vec{k}^{\prime}\right)}{\bar{k}_{b b}} \\
P_{a b}\left(\vec{k}^{\prime} \mid \vec{k}\right)=\frac{k_{a}^{\prime} P_{b}\left(\vec{k}^{\prime}\right)}{\vec{k}_{b a}} & P_{b a}\left(\vec{k}^{\prime} \mid \vec{k}\right)=\frac{k_{a}^{\prime} P_{a}\left(\vec{k}^{\prime}\right)}{\vec{k}_{a b}} .
\end{array}
$$

This implies that functions $G_{a a}(z \mid \vec{k}), G_{a b}(z \mid \vec{k}), G_{b a}(z \mid \vec{k})$, and $G_{b b}(z \mid \vec{k})$ become independent of $\vec{k}$. We further assume that the number of neighbors from $a$ and $b$ of a given node are uncorrelated, that is,

$$
P_{a}(\vec{k})=P_{a}\left(k_{a}\right) P_{a}\left(k_{b}\right) \quad P_{b}(\vec{k})=P_{b}\left(k_{a}\right) P_{b}\left(k_{b}\right) .
$$

In the case of two coupled Erdös-Rényi random graphs, the degree distributions $P_{a}\left(k_{a}\right), P_{a}\left(k_{b}\right), P_{b}\left(k_{a}\right)$, and $P_{b}\left(k_{b}\right)$ are all Poisson distributions of parameters $\bar{k}_{a a}, \bar{k}_{a b}, \bar{k}_{b a}$, and $\bar{k}_{b b}$, respectively. In this case, it is easy to check that $\hat{Q}_{a}(z)=\hat{G}_{a a}(z), \hat{Q}_{b}(z)=\hat{G}_{b b}(z)$, and

$$
\begin{aligned}
& \hat{G}_{a a}(z)=z e^{-\bar{k}_{a a} p\left(1-\hat{G}_{a a}(z)\right)} e^{-\bar{k}_{a b} p\left(1-\hat{G}_{a b}(z)\right)}, \\
& \hat{G}_{a b}(z)=z e^{-\bar{k}_{b a} p\left(1-\hat{G}_{b a}(z)\right)} e^{-\bar{k}_{b b} p\left(1-\hat{G}_{b b}(z)\right)}, \\
& \hat{G}_{b a}(z)=z e^{-\overline{-}_{a b} p\left(1-\hat{G}_{a b}(z)\right)} e^{-\bar{k}_{a a} p\left(1-\hat{G}_{a a}(z)\right)}, \\
& \hat{G}_{b b}(z)=z e^{-\bar{k}_{b b} p\left(1-\hat{G}_{b b}(z)\right)} e^{-\bar{k}_{b a} p\left(1-\hat{G}_{b a}(z)\right)} .
\end{aligned}
$$

Finally, using $g_{a}=1-\hat{Q}_{a}(z=1)=1-\hat{G}_{a a}(z=1)$ and $g_{b}=1-\hat{Q}_{b}(z=1)=1-\hat{G}_{b b}(z=1)$ and after defining $g_{a b}=1-\hat{G}_{a b}(z=1)$ and $g_{b a}=1-\hat{G}_{b a}(z=1)$, we obtain Eq. (2).

\section{APPENDIX E: FINITE-SIZE SCALING OF THE CORE-PERIPHERY RANDOM GRAPH MODEL}

In percolation theory, the susceptibility is usually evaluated by the average size of finite clusters [38]. An alternative approach is to use the fluctuations of the order parameter, with a proper normalization $[45,46]$. The advantage of this approach is that these fluctuations involve the extreme values of the cluster size distribution, which are responsible for the critical fluctuations of the process. In this work, we follow this approach but with a different normalization. In the standard version, the susceptibility is defined as

$$
\chi_{\mathrm{st}} \equiv \frac{\left\langle G^{2}\right\rangle-\langle G\rangle^{2}}{N},
$$

whereas ours is defined as

$$
\chi \equiv \frac{\left\langle G^{2}\right\rangle-\langle G\rangle^{2}}{\langle G\rangle} .
$$

The advantage of using Eq. (E2) instead of Eq. (E1) is mainly numerical. For a finite system of size $N$, the peak of the standard susceptibility near the critical point behaves as $\chi_{\mathrm{st}}^{\max } \sim N^{\gamma / \nu}$ and the average size of the giant component as $\langle G\rangle \sim N^{1-\beta / \nu}$ (in this context, $\gamma$ is not the exponent of the degree distribution but the critical exponent of the susceptibility). Therefore, our version of the susceptibility $\chi$ diverges near the critical point as $\chi \sim N^{\gamma^{\prime} / \nu}$, where $\gamma^{\prime}=\gamma+\beta$. This means that $\gamma^{\prime}>\gamma$, and thus, it is easier to measure in numerical simulations. 
(a)
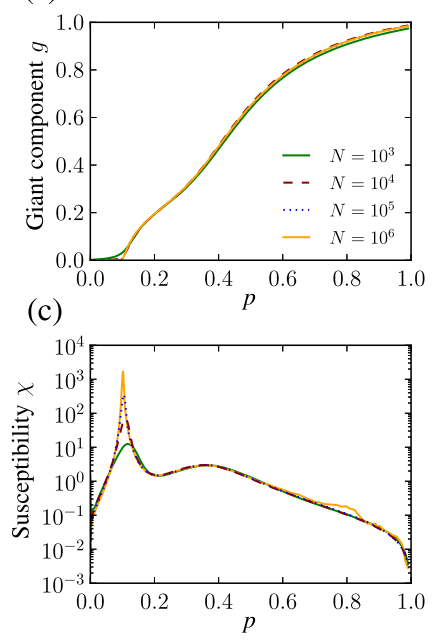

(b)

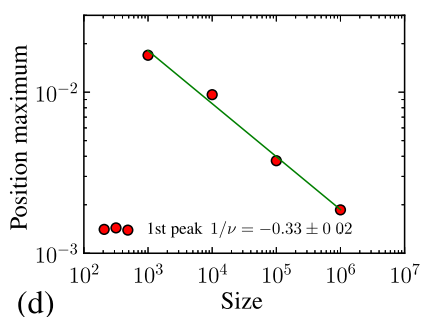

(d)

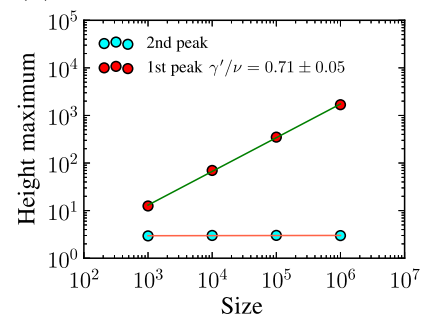

FIG. 9. Bond percolation simulations for the core-periphery random graph model for $\alpha=1$ for different sizes. In both cases, the core has an average degree $\bar{k}_{c}=10$ and the periphery $\bar{k}_{p}=2.5$. The core-periphery ratio is $r=0.2$. (a) Relative size of the largest connected component as a function of the bond occupation probability $p$. (c) Susceptibility $\chi$ as a function of bond occupation probability $p$. (b, d) Position $p_{\max }$ and height $\chi_{\max }$ of the two peaks of $\chi$ as a function of network size $N$. The straight lines are power-law fits. Panels (b) and (d) show the measured values of the critical exponents.

Let $\left(\beta_{c}, \gamma_{c}^{\prime}, \nu_{c}\right)$ and $\left(\beta_{p}, \gamma_{p}^{\prime}, \nu_{p}\right)$ be the critical exponents of the core and the periphery when they are isolated from each other. Close to the percolation transition of the core, the giant component is mainly composed of nodes in the core, and therefore, we expect the first transition to have the critical properties of regular percolation in the core subgraph; in particular, the susceptibility near the first peak diverges with the exponent $\gamma_{c}^{\prime} / \nu_{c}$. Close to the second transition point, the giant component is the sum of the giant component in the core, $G_{c}$, plus the percolating cluster in the periphery, $G_{p}$. Since $G_{c}$ and $G_{p}$ are statistically

(a)

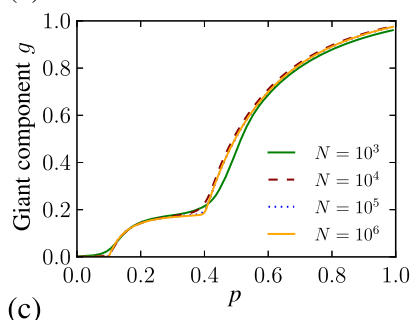

(c)

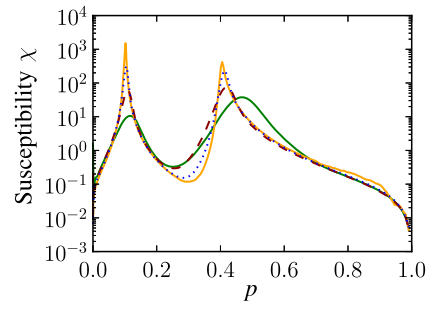

(b)
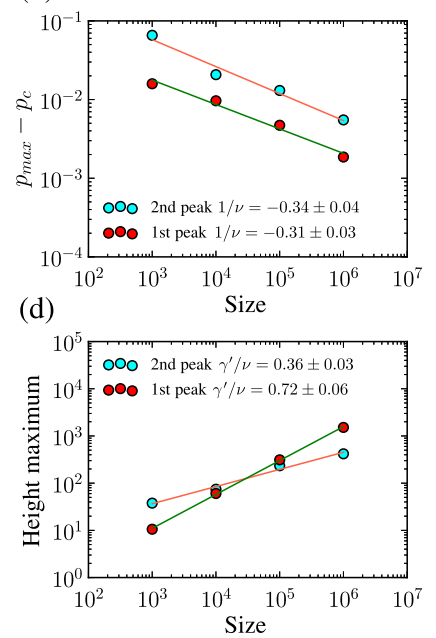

FIG. 10. Bond percolation simulations for the core-periphery random graph model for $\alpha=0.5$ for different sizes. In both cases, the core has an average degree $\bar{k}_{c}=10$ and the periphery $\bar{k}_{p}=2.5$. The core-periphery ratio is $r=0.2$. (a) Relative size of the largest connected component as a function of the bond occupation probability $p$. (c) Susceptibility $\chi$ as a function of bond occupation probability $p$. (b, d) Position $p_{\max }$ and height $\chi_{\max }$ of the two peaks of $\chi$ as a function of network size $N$. The straight lines are power-law fits. Panels (b) and (d) show the measured values of the critical exponents.

independent, the susceptibility in this region can be evaluated as

$$
\chi=\frac{\left\langle G_{c}\right\rangle \chi_{c}+\left\langle G_{p}\right\rangle \chi_{p}}{\left\langle G_{c}\right\rangle+\left\langle G_{p}\right\rangle} \approx \chi_{c}+\frac{\left\langle G_{p}\right\rangle}{\left\langle G_{c}\right\rangle} \chi_{p} .
$$

However, if the second transition point is well separated from the first one, close to this second transition $\chi_{c} \sim$ cte, $\left\langle G_{c}\right\rangle \sim N$, and $\left\langle G_{p}\right\rangle \sim N^{1-\beta_{p} / \nu_{p}}$. Then, we expect that near the second transition, the susceptibility behaves as $\chi \sim N^{\left(\gamma_{p}^{\prime}-\beta_{p}\right) / \nu_{p}}$. The critical exponents in the case of Erdös-Renyí random graphs are the mean-field ones, that
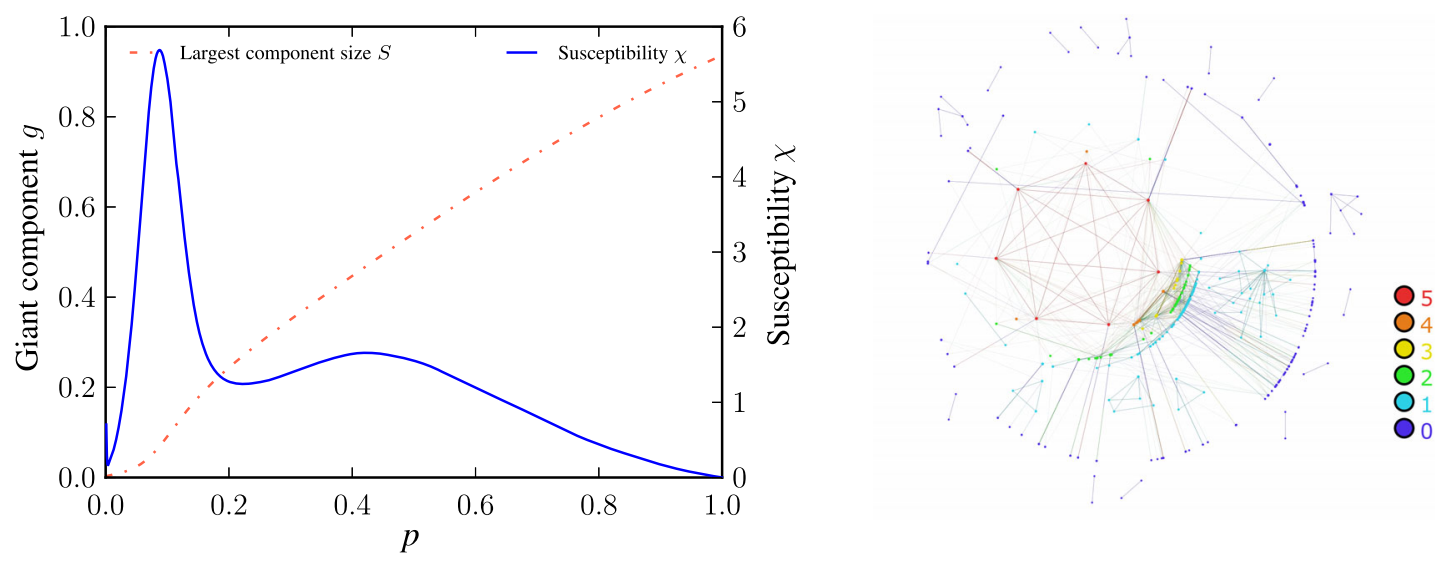

FIG. 11. Left panel: Bond percolation simulations for the US air transportation network. The relative size of the largest connected component $g$ and its susceptibility $\chi$ as a function of the bond occupation probability $p$ are shown. Right panel: $m$-core decomposition. 
is, $\beta=\gamma=1$ and $\nu=3$ [38]. Therefore, in our simulations, we expect the first peak to diverge as $N^{2 / 3}$, the second peak as $N^{1 / 3}$, and the approach of the position of the peaks to their respective critical points as $p_{\max } \sim p_{c}+A N^{-1 / 3}$. This is confirmed in Fig. 10.

\section{APPENDIX F: REAL NETWORKS}

\section{US air transportation network}

In the US air transportation network, the nodes are airports and a link is the existence of a direct flight between two airports [47]. The network has 583 nodes, 1087s edges, an average degree of $\bar{k}=3.73$, a clustering coefficient of $\bar{C}=0.43$, and a maximum degree $k_{\max }=109$. Figure 11 shows bond percolation simulations and the $m$-core decomposition of the US air transportation network.

\section{Human disease network}

In the "human disease network," nodes represent disorders, and two disorders are connected to each other if they share at least one gene in which mutations are associated with both disorders [48]. The resulting network has 867 nodes, 1527 edges, an average degree of $\bar{k}=3.52$, a clustering coefficient of $\bar{C}=0.81$, and a maximum degree $k_{\max }=50$. Figure 12 shows bond percolation simulations and the $m$-core decomposition of the human disease network.

\section{Pokec online social network}

Pokec is one of the most popular online social networks in Slovakia. Pokec has been available for more than 10 years, and it connected more than 1.6 million people by 2012. We analyze the undirected network by deleting all nonbidirectional links. For a smaller system, we only consider nodes that signed up for the online network before 2004. The resulting network has 44285 nodes, 75285 edges, an average degree of $\bar{k}=3.4$, a clustering coefficient of $\bar{C}=0.09$, and a maximum degree $k_{\max }=58$. Figure 13 shows bond percolation simulations and the $m$-core decomposition of the Pokec online social network.
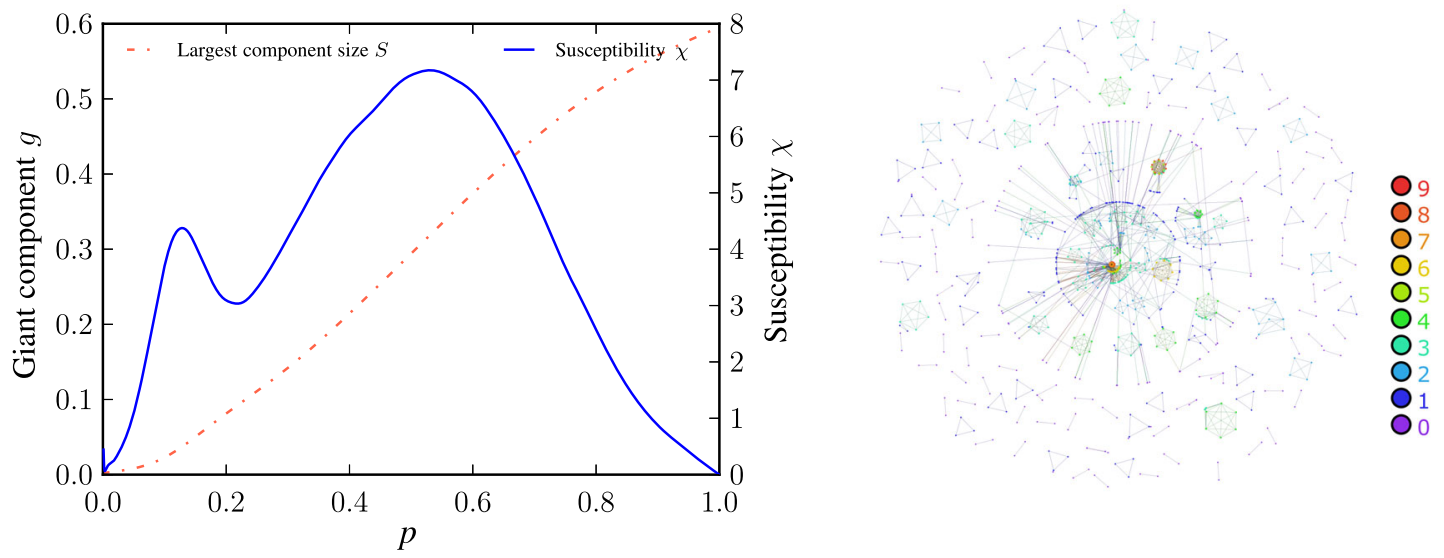

FIG. 12. Left panel: Bond percolation simulations for the human disease network. The relative size of the largest connected component $g$ and its susceptibility $\chi$ as a function of the bond occupation probability $p$. Right panel: $m$-core decomposition.
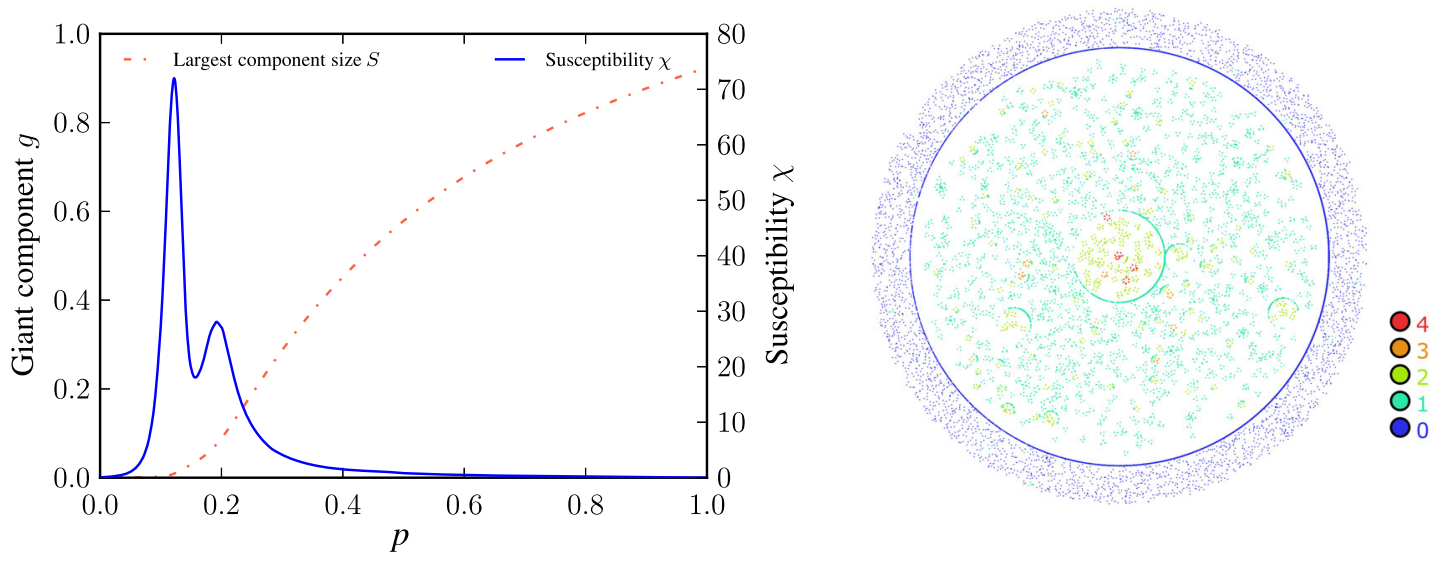

FIG. 13. Left panel: Bond percolation simulations for the Pokec online social network. The relative size of the largest connected component $g$ and its susceptibility $\chi$ as a function of the bond occupation probability $p$ are shown. Right panel: $m$-core decomposition. 
[1] R. Pastor-Satorras and A. Vespignani, Epidemic Spreading in Scale-Free Networks, Phys. Rev. Lett. 86, 3200 (2001).

[2] A. L. Lloyd and R. M. May, Epidemiology-How Viruses Spread Among Computers and People, Science 292, 1316 (2001).

[3] M. Boguna, R. Pastor-Satorras, and A. Vespignani, Absence of Epidemic Threshold in Scale-Free Networks with Degree Correlations, Phys. Rev. Lett. 90, 028701 (2003).

[4] N. Berger, C. Borgs, J. T. Chayes, and A. Saberi, On the Spread of Viruses on the Internet, in Proceedings of the Sixteenth Annual ACM-SIAM Symposium on Discrete Algorithms, SODA '05 (Society for Industrial and Applied Mathematics, Philadelphia, PA, 2005), pp. 301-310.

[5] S. Chatterjee and R. Durrett, Contact Processes on Random Graphs with Power Law Degree Distributions Have Critical Value O, Ann. Prob. 37, 2332 (2009).

[6] M. Boguñá, C. Castellano, and R. Pastor-Satorras, Nature of the Epidemic Threshold for the Susceptible-InfectedSusceptible Dynamics in Networks, Phys. Rev. Lett. 111, 068701 (2013).

[7] G. Bianconi, Mean Field Solution of the Ising Model on a Barabasi-Albert Network, Phys. Lett. A 303, 166 (2002).

[8] A. V. Goltsev, S. N. Dorogovtsev, and J. F. F. Mendes, Critical Phenomena in Networks, Phys. Rev. E 67. 026123 (2003).

[9] M. Hinczewski and A. Nihat Berker, Inverted BerezinskiiKosterlitz-Thouless Singularity and High-Temperature Algebraic Order in an Ising Model on a Scale-Free Hierarchical-Lattice Small-World Network, Phys. Rev. E 73, 066126 (2006).

[10] S. N. Dorogovtsev, A. V. Goltsev, and J. F. F. Mendes, Critical Phenomena in Complex Networks, Rev. Mod. Phys. 80, 1275 (2008).

[11] R. Cohen, K. Erez, D. Ben-Avraham, and S. Havlin, Resilience of the Internet to Random Breakdowns, Phys. Rev. Lett. 85, 4626 (2000).

[12] D. S. Callaway, M. E. Newman, S. H. Strogatz, and D. J. Watts, Network Robustness and Fragility: Percolation on Random Graphs, Phys. Rev. Lett. 85, 5468 (2000).

[13] R. Cohen, D. Ben-Avraham, and S. Havlin, Percolation Critical Exponents in Scale-Free Networks, Phys. Rev. E 66, 036113 (2002).

[14] M. Newman, Assortative Mixing in Networks, Phys. Rev. Lett. 89, 208701 (2002).

[15] M. E. J. Newman, Properties of Highly Clustered Networks, Phys. Rev. E 68, 026121 (2003).

[16] A. Vázquez and Y. Moreno, Resilience to Damage of Graphs with Degree Correlations, Phys. Rev. E 67, 015101 (2003).

[17] S. N. Dorogovtsev, J. F. F. Mendes, and A. N. Samukhin, Anomalous Percolation Properties of Growing Networks, Phys. Rev. E 64, 066110 (2001).

[18] D. S. Callaway, J. E. Hopcroft, J. M. Kleinberg, M. E. J. Newman, and S. H. Strogatz, Are Randomly Grown Graphs Really Random?, Phys. Rev. E 64, 041902 (2001).

[19] S. V. Buldyrev, R. Parshani, G. Paul, H. Eugene Stanley, and S. Havlin, Catastrophic Cascade of Failures in Interdependent Networks, Nature (London) 464, 1025 (2010).

[20] S.-W. Son, G. Bizhani, C. Christensen, P. Grassberger, and M. Paczuski, Percolation Theory on Interdependent
Networks Based on Epidemic Spreading, Europhys. Lett. 97, 16006 (2012).

[21] G. J. Baxter, S. N. Dorogovtsev, A. V. Goltsev, and J. F. F. Mendes, Avalanche Collapse of Interdependent Networks, Phys. Rev. Lett. 109, 248701 (2012).

[22] M. Newman, Properties of Highly Clustered Networks, Phys. Rev. E 68, 026121 (2003).

[23] M. Serrano and M. Boguñá, Clustering in Complex Networks. II. Percolation Properties, Phys. Rev. E 74, 056115 (2006).

[24] I. Kiss and D. Green, Comment on "Properties of Highly Clustered Networks,” Phys. Rev. E 78, 048101 (2008).

[25] M. Newman, Random Graphs with Clustering, Phys. Rev. Lett. 103, 058701 (2009).

[26] J. Miller, Percolation and Epidemics in Random Clustered Networks, Phys. Rev. E 80, 020901 (2009).

[27] J. Gleeson, Bond Percolation on a Class of Clustered Random Networks, Phys. Rev. E 80, 036107 (2009).

[28] J. P. Gleeson, S. Melnik, and A. Hackett, How Clustering Affects the Bond Percolation Threshold in Complex Networks, Phys. Rev. E 81, 066114 (2010).

[29] P. Csermely, A. London, L.-Y. Wu, and B. Uzzi, Structure and Dynamics of Core-Periphery Networks, J. Complex Networks 1, 93 (2013).

[30] J. Nagler, T. Tiessen, and H. W. Gutch, Continuous Percolation with Discontinuities, Phys. Rev. X 2, 031009 (2012).

[31] W. Chen, J. Nagler, X. Cheng, X. Jin, H. Shen, Z. Zheng, and R. M. D'Souza, Phase Transitions in Supercritical Explosive Percolation, Phys. Rev. E 87, 052130 (2013).

[32] W. Chen, X. Cheng, Z. Zheng, N. Ning Chung, R. M. D'Souza, and J. Nagler, Unstable Supercritical Discontinuous Percolation Transitions, Phys. Rev. E 88, 042152 (2013).

[33] G. Bianconi and S. N. Dorogovtsev, Multiple Percolation Transitions in a Configuration Model of Network of Networks, Phys. Rev. E 89, 062814 (2014).

[34] Note that this case is not the same as fixing the average clustering coefficient because, in our case, we enforce nodes of any degree to have the same local clustering. In any case, due to structural constraints, for very strong clustering it is not possible to keep $\bar{c}(k)$ constant for very large values of $k$. In this case, the algorithm generates the maximum possible clustering [35].

[35] M. A. Serrano and M. Boguñá, Clustering in Complex Networks. I. General Formalism, Phys. Rev. E 74, 056114 (2006).

[36] M.E. Newman and R. M. Ziff, Efficient Monte Carlo Algorithm and High-Precision Results for Percolation, Phys. Rev. Lett. 85, 4104 (2000).

[37] Note that we evaluate the size dependence as a function of the total number of nodes $N$ and not as the one-dimensional length $L$. This implies that the finite-size critical exponent $\nu$ that we use in this paper already includes the dimensionality of the system $d$; that is, our exponent $\nu$ is equivalent to $d \nu$ in [38].

[38] D. Stauffer and A. Aharony, Introduction to Percolation Theory (CRC Press, London, 1994).

[39] P. Colomer-de Simón, M. Ángeles Serrano, M. G. Beiró, J. Ignacio Alvarez-Hamelin, and M. Boguñá, Deciphering the 
Global Organization of Clustering in Real Complex Networks, Sci. Rep. 3, 2517 (2013).

[40] D. Strauss, On a General Class of Models for Interaction, SIAM Rev. 28, 513 (1986).

[41] J. Park and M. Newman, Solution for the Properties of a Clustered Network, Phys. Rev. E 72, 026136 (2005).

[42] D. Foster, J. Foster, M. Paczuski, and P. Grassberger, Communities, Clustering Phase Transitions, and Hysteresis: Pitfalls in Constructing Network Ensembles, Phys. Rev. E 81, 046115 (2010).

[43] S. Melnik, M. A. Porter, P. J. Mucha, and J. P. Gleeson, Dynamics on Modular Networks with Heterogeneous Correlations, Chaos 24, 023106 (2014).
[44] R. Milo, S. Shen-Orr, S. Itzkovitz, N. Kashtan, D. Chklovskii, and U. Alon, Network Motifs: Simple Building Blocks of Complex Networks, Science 298, 824 (2002).

[45] M. E. J. Newman and G. T. Barkema, Monte Carlo Methods in Statistical Physics (Clarendon Press, Oxford, 1999).

[46] J. Marro and R. Dickman, Nonequilibrium Phase Transitions in Lattice Models (Cambridge University Press, Cambridge, England, 1999).

[47] M. Ángeles Serrano, M. Boguñá, and A. Vespignani, Extracting the Multiscale Backbone of Complex Weighted Networks, Proc. Natl. Acad. Sci. U.S.A. 106, 6483 (2009).

[48] K.-I. Goh, M. E. Cusick, D. Valle, B. Childs, M. Vidal, and A.-L. Barabási, The Human Disease Network, Proc. Natl. Acad. Sci. U.S.A. 104, 8685 (2007). 